\title{
Service Interoperability through advanced \\ Media Gateways
}

\author{
Sven van der Meer, Stefan Arbanowski \\ Technical University Berlin, Franklinstrasse 28/29, 10587 Berlin, Germany \\ Email: vdmeer@cs.tu-berlin.de \\ Tel: +49 303463 7158, Fax: Tel: +49 3034638158
}

The convergence of telecommunications systems and the Internet causes a variety of concepts for service integration. The focus of the recent research studies and the work of several standardization bodies lies, mostly, on the interworking of services and the universal service access from end-user systems including both, fixed and wireless terminals. All approaches are driven by the concept of providing several technologies to users by keeping the peculiarity of each service alive.

But, developments should not only concentrate on media adaptation between VoIP and PSTN, but also consider the adaptation among completely different types of applications as for example email, fax, or voice. Unified Messaging, which is an already accepted service on the market, provides solutions for conversions of different application protocols into each other. The functionality of converting one media into another is implemented here in so called Media Gateways.

This paper provides an overview of the current developments in the area of Media Gateways. Next, it introduces the basic architecture of an existing, CORBA-based unified messaging system and the implementation of Media Gateways for the adaptation of different services. Finally, both approaches are combined to Unified Messaging enabled Media Gateways that improve the user's reachability because of their capability to adapt services to different kinds of end devices.

Keywords: Media Gateways, Service Personalization, Service Adaptation, Unified Messaging, Media Conversion, IP, User Mobility, UMTS, VHE, VoIP

\section{Introduction}

Internet technologies have proven their applicability to the future telecommunications environment. Emerging architectures for Internet Telephony, such as the ITU-T H.323 standard [1] or the IETF drafts of MGCP [2] and Megaco [3], offer solutions for the integration of the telecommunication

The original version of this chapter was revised: The copyright line was incorrect. This has been corrected. The Erratum to this chapter is available at DOI: 10.1007/978-0-387-35522-1_37 
world and the Internet. However, these activities concentrate mainly on the provision of a basic voice communication service, leaving value-added services (like Intelligent Network (IN) services) out of scope. New developments try to combine VoIP services with the IN, resulting on so called soft switches able to process IN-like services over IP networks. In this context, a Gatekeeper or a Media Gateway Controller takes over the signaling and Media Gateways are responsible for the adaptation of transport protocols.

The concept of Unified Messaging has recently emerged from the research of Personal Communication Support (PCS). Reaching industrial relevance, it addresses the task of overcoming the multiple-mailbox approach of today's messaging systems, with separated facilities for e-mail, voice storage, fax reception, etc. This coincides with the vision for future communication, to deliver information any time, any place, in any form, as it is described in the Virtual Home Environment (VHE) concept within the Universal Mobile Telecommunication System (UMTS) standards. [4] [5]

Messaging systems take into account new application fields such as locationawareness, terminal-awareness, and context-awareness. This means, that the user is supported by the seamless integration of new network access technologies (e.g. UMTS), new terminal equipment (e.g. Personal Digital Assistants and enhanced mobile phones), and new multimedia applications. The more such technologies are in common use, the more the user's communication behavior will change. The main interest of the users is no longer the ubiquitous access to their messages but the control of their reachability. Messaging systems have to support the user to define exactly when, where, and for whom he is reachable. This means that people will define their own individual communication and services environment, specifying how they want to use services and in which way.

The restricted capabilities of a terminal limit users in being reachable for different kind of services. To overcome this restriction, messaging systems adapt one service to another employing conversion technologies. The system provides the user the capability to configure service-interworking scenarios for specific types of messages and invokes the appropriate conversion to deliver the received messages. The evaluation of an appropriate conversion strategy applies application based Quality of Service (QoS) preferences made by the user. But, users are not interested to define technical QoS parameters like jitter, delay, or bandwidth. Most users even do not understand the technological background of them. Users can describe their preferences by i.e. means of costs of delivery and intelligibility of converted information.

One prerequisite for the vision of information any time, any place, in any form is global connectivity, based on a fast developing web of interconnected communication networks, comprising fixed and wireless networks as well as the Internet (figure 1). In addition, the provision of a global service infrastructure, based on network-independent open service platforms is the other fundamental prerequisite, hiding the complexity of network diversity. This 
platform allows the fast and efficient creation, provision, and management of services.

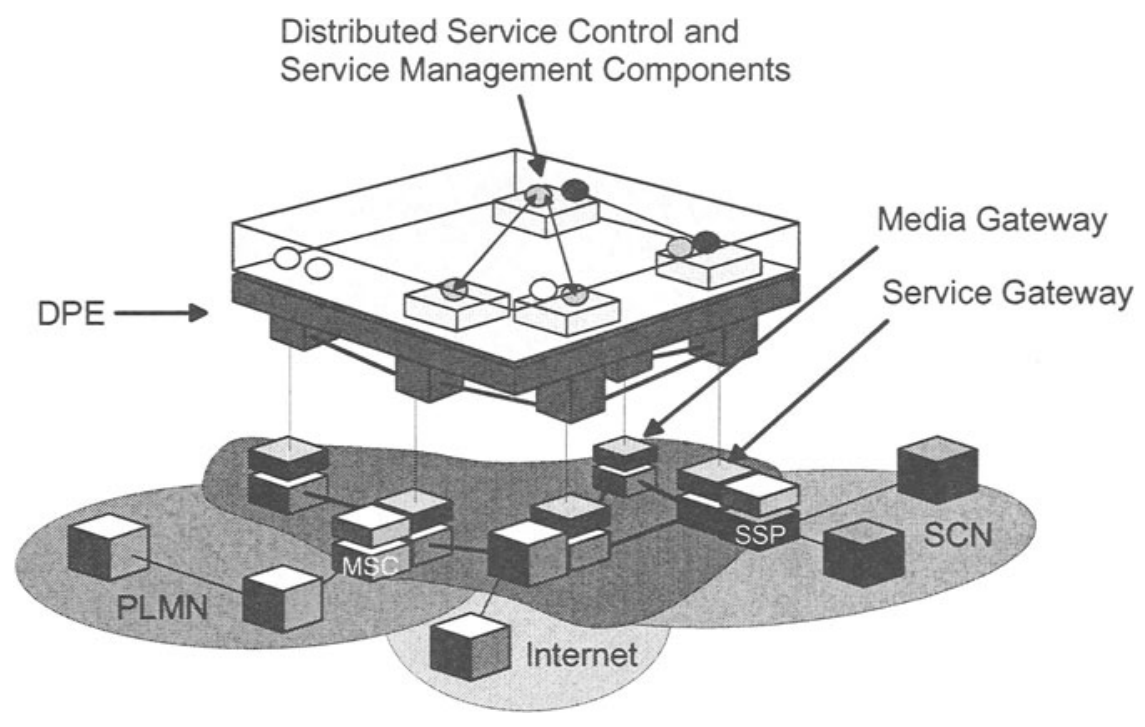

Figure 1: Future Telecommunications Environment

The spectrum of services ranges from simple communication services up to complex distributed applications. In particular, intelligent information and messaging services are commonplace. Additionally, services providing information retrieval (news on demand, brokerage information on demand), services emerging from the field of electronic commerce (hotel or flight reservation), and services like the intelligent house are gaining momentum.

With regard to such services and in addition to the understanding of different standards of Media Gateways, the developments should not only concentrate on media adaptation as for example between VoIP and PSTN, but also consider the adaptation among completely different types of applications as for example email, fax, or speech. To analyze such kind of adaptation, the concept of Unified Messaging, which is an already accepted service on the market, provides solutions for conversions of different application protocols into each other.

\section{VoIP Media Gateways - Current Situation}

Fundamentally, Gateways are used for connecting different networks. If these networks are of the same type, the gateway simply performs the routing of information between them almost without any adaptation. This relates for example to the Internet Protocol where the gateways are responsible for the forwarding of IP packets to other subnetworks. However, in order to enable the interconnection of different kinds of networks, gateways also have to provide an adaptation to the respective transport media and protocols. 
The more interesting and today still topical aspects regard to the interworking of services that are provided in different networks. The IP telephony is a well-known example for this. The VoIP applications shall be able to establish connections to legacy telephone devices and vice versa. Here, there is on the one side the packet orientated Internet and on the other side a Switched Circuit Network (SCN) like PSTN/ISDN. The later is traditionally used for the telephony service. With the continual development of the Internet, the multimedia communication was given an important role. In 1995, the first Internet Telephony software was developed by Vocaltec corporation. The H.323 recommendation is the principal ITU-T standard for Internet telephony. It is an umbrella standard defining a protocol framework including several specification concerning the signaling, coding, and transmission of multimedia data over the Internet protocol.

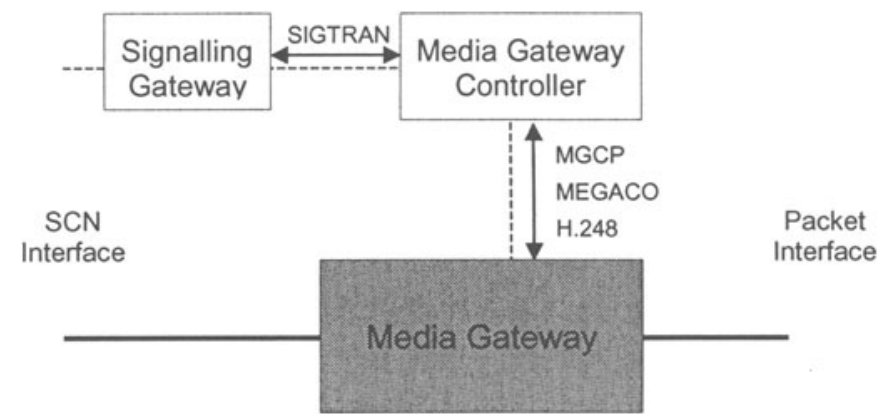

Figure 2: General Architecture of Media Gateways

Whereas PSTN is strongly established worldwide, IP telephony is to a certain point of degree still topic of research and development but it has huge potential to revolutionize the world of telecommunication and is a serious challenge to PSTN. There are still problems to be solved, like the paradigm 'always on' feasible for any common telephone or the immediate delivery of an emergency call. Other questions are security issues of telephony equipment with an IP address that is connected to the internet. Such hardware can easily be controlled remotely or can be interfered with denial of service attacks. To become successful finally, the interworking to traditional telephony must be made possible. This leaded to the introduction of Media Gateways.

TIPHON ("Telecommunications and Internet Protocol Harmonization Over Networks") is an ETSI project that has defined a distributed architecture for realization of such gateways between IP-based Internet and PSTN. This decomposed architecture is based on three components - Media Gateway, Media Gateway Controller, and Signaling Gateway (figure 2). Because of the distribution, the necessity for standardized interfaces and protocols arose. Several standardization forums such as ITU-T and IETF have been working in this. TIPHON considers exemplary a couple of Media Gateways for different kinds of applications in [9], including: 
- Trunk gateways that interface between SCN networks and IP networks. Such gateways typically interface to SS7 or other NNI signaling on the SCN and manage a large number of digital circuits.

- Access gateways that interface UNI interfaces like ISDN (PRI and BRI) and traditional analogue interfaces to a Voice over IP network.

- Residential gateways are access gateways for a small number of voice terminals that can be co-located with a cable modem or set top box.

- Network Access Servers, which convert modem signals from an SCN network and provide data access to the packet network.

- Interactive Voice Response systems that provide automatic voice response and switching services in response to DTMF signals from the SCN.

- IP Gateways that are used to interface either between administrative domains which apply different policies (e.g. proxies), or to transform media streams formats (e.g. transcoding).

Originally developed for the interconnection of different types of networks, gateways in form of Media Gateways are today increasingly deployed to enable the interworking of services.

In this section, the concept of media gateways was introduced with media gateways as the intermediate components that enable the interconnection of different networks and the interworking of similar services. The next consequent step in this evolution will be the enhancement of media gateways to enable a very capable interworking of different kind of services.

\section{Media Gateways \& Unified Messaging}

The idea of media gateways in unified messaging systems is to establish an intelligent control of these conversion capabilities by mapping user preferences to actual system behavior. This means, a media gateway converts a specific message according to user or systems demands. User demands are taken from profiles, whereas system demands result from network related aspects (e.g. the media gateway has to adapt an incoming ISDN call to the GSM world, because the callee is using a cellular phone).

The department for Open Communication Systems (OKS) at the Technical University Berlin has developed a 4th generation Unified Messaging System (UMS) [10]. The focus of the system lies on the interworking and integration of legacy and future communication services, such as telephony, voicemail, fax, email, paging, Tele-conferencing, and IP-Telephony. It offers a universal access from a variety of end user systems, including both fixed and mobile terminals. The common attribute is the notion of customer control / customer 
profile management enabling users to define rules for the handling of incoming calls and messages in accord to their individual preferences. [Vdm99a]

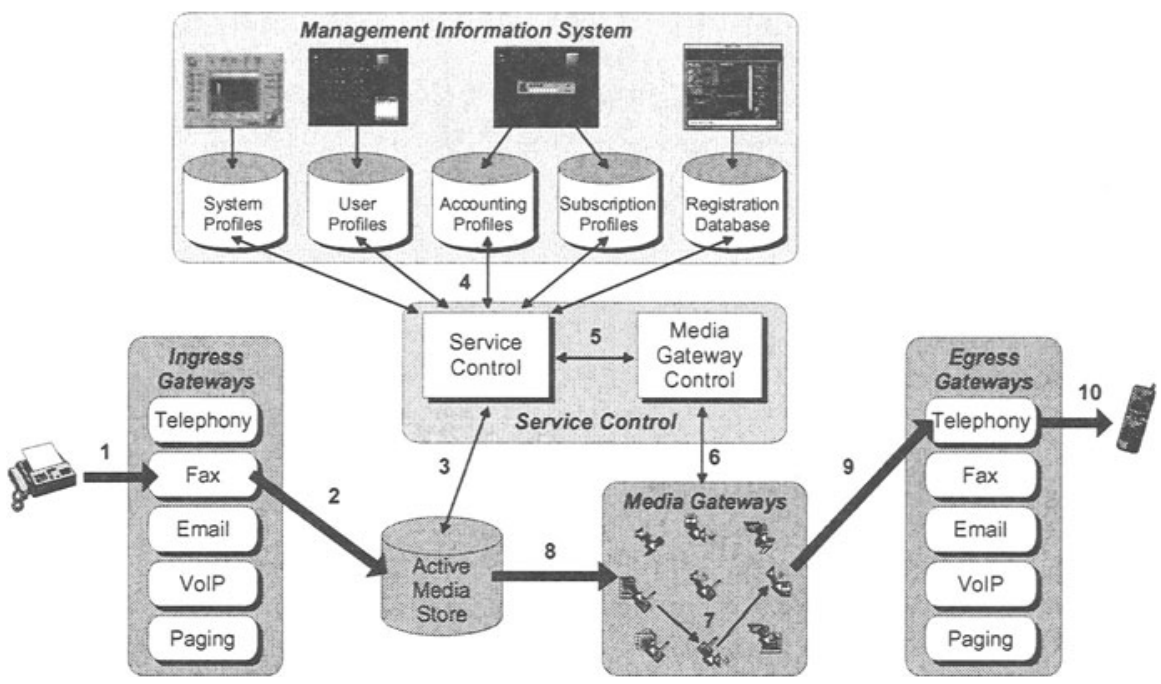

Figure 3: UMS - Functional Components

Typical scenarios featured by UMS are the support of mobile users with universal access to their services. A special capability is the selection of the most appropriate terminal or application for the delivery of an incoming message. Furthermore, the system can adapt any kind of services by conversion processes, e.g. to deliver an incoming e-mail via Short Messaging Service to a mobile phone. UMS provides features like:

- Call Screening/Scheduling/Forwarding,

- Customer Profile Management,

- Media Conversion, Content Screening/Handling,

- Multimedia Message Storage, and

- Automatically/Manually User Registration at locations or terminals.

\subsection{Functional Components}

The functional components of UMS and an example of their interworking based on the forwarding of a fax message to a telephone are given in Figure 3.

1) The Ingress Service Gateways connect the messaging system to SCNs and the Internet. They can be associated to synchronous and asynchronous services. Asynchronous services, also known as store and forward services, receive information in form of messages. After the complete reception of a message, it is handed over to the Active Media Store. 
2) The Active Media Store provides persistent storage capabilities of multimedia information and universal access to them. After receiving the fax message from the gateway, it processes the content of the fax to collect all information for Service Control. E.g., to select an appropriate conversion strategy it is necessary to extract the text out of the fax image and to identify the language the text is written in. Because a conversion of a message always means a loss of information, the system is not going to remove this stored message, unless it is requested to do so by the user.

3) Service Control is the service logic of the messaging system. It queries other components (4) to get on-demand information on user/system preferences. It finds out where a user is located at, which communication capabilities he can use, which services he is permitted to use, and when, for whom, in which way the user wants to be reachable. This information is passed on to Media Gateway Control.

$5,6,7,8,9)$ Media Gateway Control controls both, the conversion process and the stream binding between resources. This comprises the instantiation, configuration, start-up, suspension, reconfiguring, restart, termination, and cleanup of any kind of software/hardware realized within the pool of Media Gateways. We have defined a unified interface to access the different kinds of Media Gateway.

10) Finally, the fax message is read out to a telephone line.

\subsection{Media Conversion}

Conversion technology reached a state, where it can be used in communication systems to adapt services to limited terminal capabilities. The so called media converters may be defined as a system entity, which input is information I1, with the semantic S1, carried by a specific medium M1, using a specific format $\mathrm{F} 1$. The media converter will distribute information $\mathrm{I} 2$ as output in medium M2, format F2, carrying semantic S2. The quality of the conversion can be measured by comparing S1 and S2. Generally, two major classes of conversions can be identified. [11]

Media Format conversions change the format the information is coded in or modifies specific parameters of the media format, e.g. converting a GIF coded image to a JPG coded image or changing the scaling of a picture. Such converters are expected to be highly flexible, allowing most possible conversion combinations.

Media Type conversion alters the type of the medium (M1 to M2). The term medium is related to the human senses. E.g., these converters are able to transform an image containing characters (like the received fax) into spoken words. Because formats of images differ from formats of voice streams, they have to be converted, too. A media conversion is specialized to a specific task, e.g. Optical Character Recognition (OCR) or Text-To-Speech (TTS) 
conversion. The accepted input formats and the produced output formats are limited.

According to the fixed amount of supported input and output formats, media type converters have to be accompanied with media format converters. This leads to a large range of supported media formats for a single media type conversion. The result is a framework of media type converters and media format converters. This framework can be seen as a single and flexible new converter. Inside of this framework, messages have to be stored temporarily (storage dimensions depends on the supported services) and stream binding between the implemented converters has to be supervised (but can be done in a homogeneous environment using proprietary technologies).

For the conversion of messages or communication services, media converters have to be chained to so called converter chains. To illustrate this, the following example shows the steps necessary to read out the fax to a telephone device:

1) analyze the fax to gain information about the content. Result should be a decision, if the fax images contains text or images, and the language of the probably included text;

2) convert the image via an OCR to plain text;

3) preprocess the text to reformat it (for increase of the intelligibility), to remove unnecessary information, and to adapt it to a coding format understood by the TTS conversion;

4) alter the text to speech by a TTS conversion. Output is a stream of audio data;

5) change the format of the audio stream to a format, which can be read out to a telephone (e.g. WAV to $\mu$ law);

This particular service adaptation needs five different converters. All of these converters have to be started, parameterized, activated, deactivated, and finally terminated. During the activation of the converters, the data has to be streamed from one converter to the next. Streams have to be bound between the input and output interfaces of pairs of converters and supervised to guarantee the correct transmission of data. In our implementation, we analyze the actual language the text is written in on a paragraph basis. So all conversion steps after the OCR can be done for single paragraph written in different languages.

For implementing such kinds of media gateways the industry provides offthe-shelves products, like automatic speech recognition, optical character recognition, interactive voice response, speech synthesis, text-to-speech conversion, language detection, text filtering, content analysis, etc. These products are combined to a powerful conversion environment, which is able to adapt any media type to any other. 


\section{Advanced Media Gateways for Interoperability of Services}

Facing the functionality of Unified Messaging Systems, the demand for a variety of conversion technology is obvious. In general, such systems provide functionality to convert email, voicemail, facsimile, and short messages into each other. These conversion processes are executed on certain media converters that could be seen as media gateways. They are capable to convert a specific medium type or media format into another one.

The concept of Media Gateways in the understanding of different standardization bodies such as ITU and IETF concentrates actually on media adaptation between VoIP and PSTN. In the area of Unified Messaging, such gateways are also responsible for the conversion of media format and media type keeping the semantic of the message intact. Such conversion of service data depends not on the transport network. It is essential for the integration of asynchronous messaging services to differ between the actual signaling of a service and the representation of the streaming of the service data. For instance, a Text-to-Speech conversion can be handled in the same way for each kind of text, regardless the original service like fax or email. This provides a service independent handling of service data for the conversion between services like show in figure 4.

Multiple or chained conversions may result in several drawbacks. The first is the delay introduced through the conversion process depending on the computing power of the employed hardware platform. The second is the loss of information within the converted message. No conversion process can be done without the loss of information. The question is, how this information loss can be described, measured, and configured by the user. We have implemented an application based QoS evaluation mechanism that provides this functionality (for details see [11]).

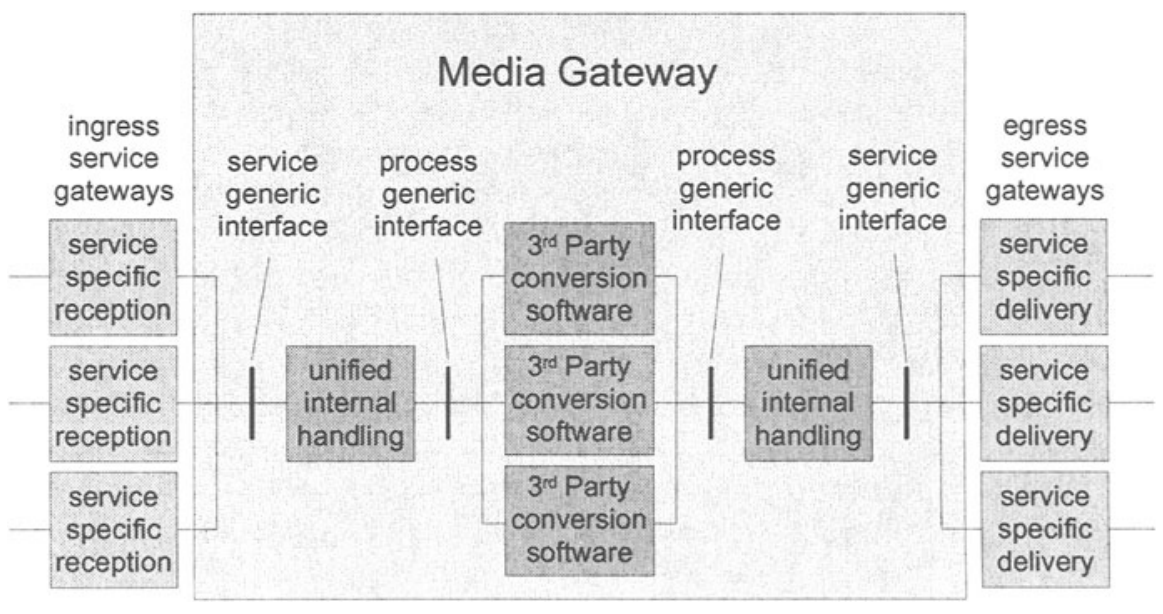

Figure 4: Media Gateways for Service Interoperability 
The proposed solution is to distinguish between Service Gateways that provide the access to various transport networks and specific service signaling and Media Gateways that realize the adaptation of service data. A set of distributed components grants the evaluation of the user's preferences and the selection of appropriate conversion strategies on top of these two kinds of gateways.

\subsection{Call Processing}

The opportunities of Media Gateways impact the call processing of telecommunication systems and the information contained in user profiles:

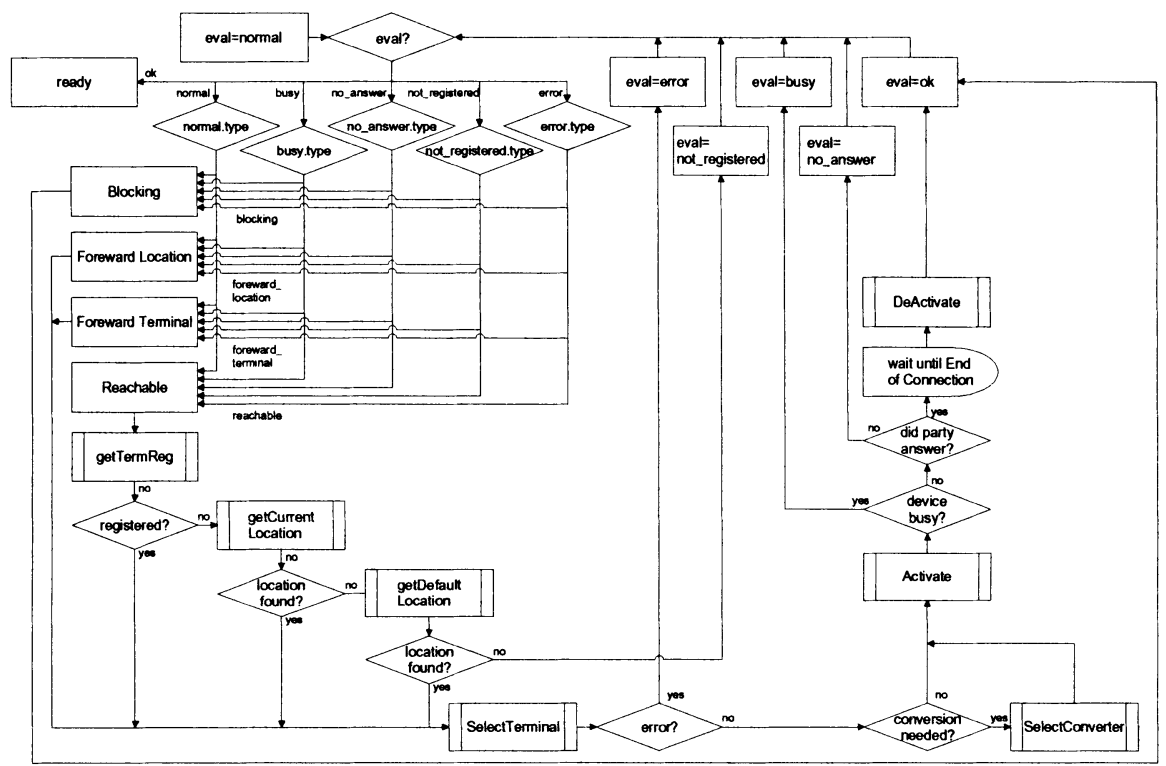

Figure 5: Call Processing Logic

All user preferences necessary for the call processing are stored in a rule based customer service profile. Rules define conditions on which several actions have to be processed (e.g. from whom the message is sent and at what time, the terminal a user is currently registered at, or the type of messaging service, the message is transported by). Possible actions for the call processing are blocking the call (blocking), to forward the call to a specific terminal (forward terminal), to forward the call to a specific location (forward location), or on-demand evaluation of the user's registration (reachable). Each action can be coupled with additional information, like forced/forbidden services. The execution of a rule can result in numerous states; normal delivery (normal), busy terminal (busy), user does not answer (no answer), user is actually not registered (not registered), or various cases of failures (error). The user can define the system behavior by specifying a rule for each state.

The resulting call processing (figure 5) is not trivial. The evaluation begins with the state normal. If a valid rule for this state can be found in the user 
profiles, the related action will be executed. The actions can be blocking, forwarding, or reachable. In case the action is set to reachable, the call processing has to request further information from the user's profile. If the user is registered at a specific terminal (e.g. a mobile phone), the call should be delivered to that terminal. If the user is registered at a specific location (inhouse via active badge systems, via GSM cell detection, or via GPS), the terminals available at this location have to be identified and the most appropriate terminal is selected for the delivery. If the finally selected terminal is not able to receive the call directly (e.g. fax to phone), a suitable Media Gateway has to be chosen. Then, the call can be delivered with appropriate media conversion.

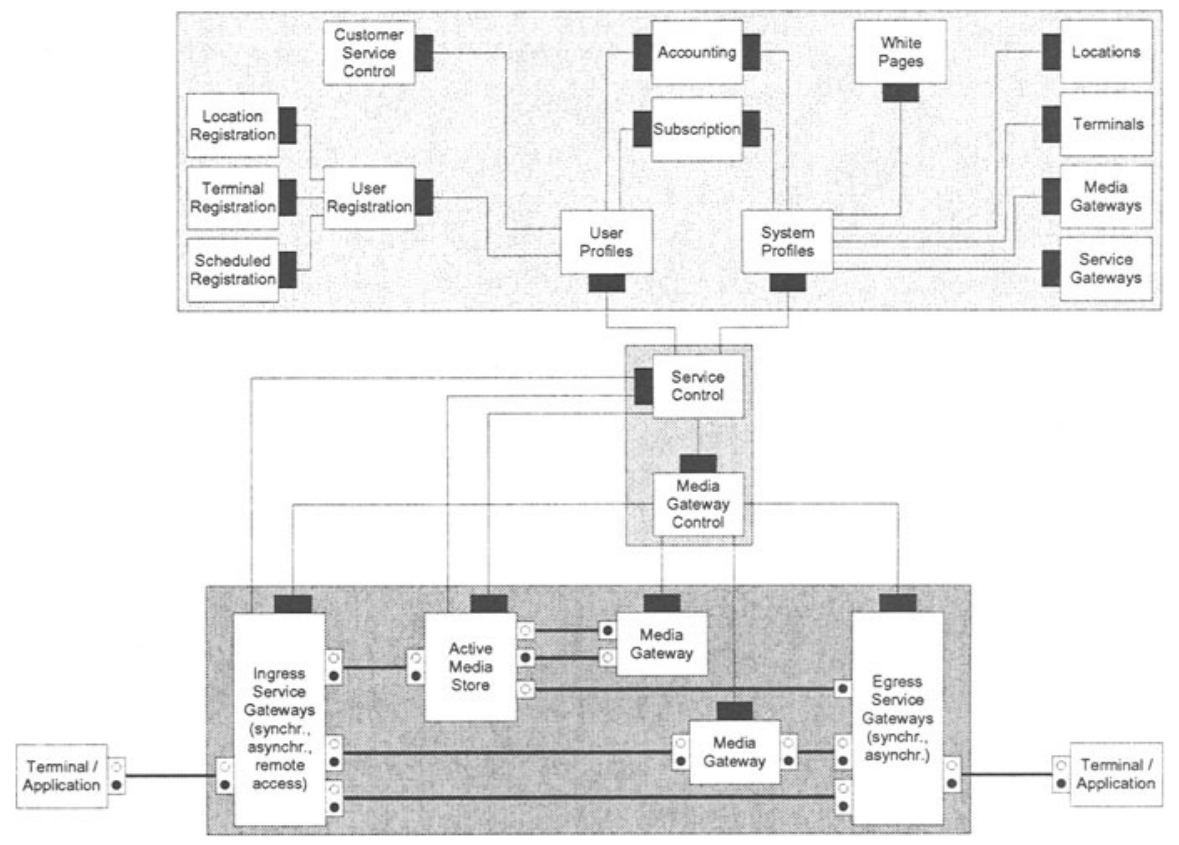

Figure 6: Distributed Components

During the delivery of the call, the system can run into other states. The terminal is busy or the user does not answer. Then, the call processing is invoked again and will now evaluate the rules specified for the new state. Therefore, mechanisms have to be provided to prevent internal loops and other failures.

Please note, that this call processing has been developed for the design and the implementation of our UMS system and the control of our Media Gateways. The evaluation of a possible adaptation to UMTS and 3GPP developments is still in progress. 


\subsection{System Architecture}

The messaging system architecture comprises a certain number of distributed objects, representing different functional blocks of the system as introduced in section 3.1 and shown as computational objects in figure 6. Each of them has a well defined interface which offers its functionality to other objects. In the following these functional blocks are called components. A detailed description of each component can be found in [10].

The intention to subdivide the system into components results from the requirement of a freely scalable and distributed system. In a component based architecture it is possible to substitute a component by an extended one, without affecting the rest of the system. A future extension could be made easily. The only precondition are carefully designed interfaces between the object, which should be unchanged during the implementation process.

The components can be grouped in to three main blocks. The lowest block consists of all objects which are responsible for streaming and processing of service data. This includes the Service Gateways and the Media Gateways. The middle block represents service logic, service control and the media gateway control.

The implementation covers all components shown in figure 6. The system itself is up and running since November 1999. After a phase of intensive testing by our development team, a first real-life version is going to be installed at the end of May 2000.

For the realization of our system we use Orbix 2.3 (including Name Service), a CORBA 2.0 conform implementation. All components are realized on Windows NT using Microsoft's Visual C++ 6.0. The core implementation is based on Rogue Wave's Tools.h++ 8.0 Professional library package.

\section{Summary}

his paper described an approach, how Media Gateways currently used for Internet Telephony can be enhanced with the capability of media conversion. The basic concepts for this enhancement have been developed in the area of Unified Messaging, where the conversion of different medias is used for the interoperability of applications.

One central point of our approach is the distinction between a Service Gateway and a Media Gateway. Service Gateways are responsible for service specific signaling, whereas Media Gateways are responsible for the actual conversion of the content of the services. This provides an easy integration of new services, because the conversion process does not rely on the service signaling. On the other hand, new conversion technologies can be applied to the system without affecting the supported communication services. 
The described approach and the already implemented system are currently analyzed in several European projects with regard to existing standards and working groups, namely the 3GPP, ITU-T, and IETF.

\section{References}

[1] ITU-T Rec. H.323, Packet-based multimedia communications systems, 1998

[2] Greene, N., Ramalho, M., Rosen, B.: Media Gateway control protocol architecture and requirements. IETF Internet draft, September 1999

[3] Cuervo, F., Huitema, C., Kelly, K., Rosen, B., Sijben, P., Zimmerer, E.: MEGACO Protocol, IETF Internet draft, July 1999

[4] ETSI Techn. specification TS 22.01v.3.1.0: Universal Mobile Telecommunication Systems (UMTS). Aspects, Principles. - Sophia Antapolis, France, 1997

[5] ETSI Draft 22.70v.0.0.3: Virtual Home Environments. - Sophia Antapolis, France, 1997

[6] Lennox, J., Schulzrinne, H., La Porta, T.: Implementing Intelligent Network Services with the Session Initiation Protocol. Tech-Report Number CUCS002-99

[7] Magdanz, T., et.al.: Towards an Integrated Architecture for the Harmonisation of PSTN and Internet Services. International Conference on Intelligence in Networks, ICIN 2000, Bordeaux, France, January 18-20, 2000

[8] Zizza, F., et.al.: Towards a Distributed Intelligent Network. International Conference on Intelligence in Networks, ICIN 2000, Bordeaux, France, January 18-20, 2000

[9] ETSI TIPHON Homepage: http://www.etsi.org/tiphon

[10] van der Meer, S.; Arbanowski, St.: An Approach for a 4th Generation Messaging System. Proc. of the 4th International Symposium on Autonomous Decentralized Systems, ISADS'99, Tokyo, Japan, 1999, 158-167

[11] Pfeifer, T.; van der Meer, S.: The Active Store providing Quality Enhanced Unified Messaging. Proc. of the 5th Conference on Computer Communications, AFRICOM-CCDC’98, Tunis, October 20-22-1998 